\title{
The association of the lipidomic profile with features of polycystic ovary syndrome
}

\author{
L J Moran1,2, P A Mundra3, H J Teede1,4,* and P J Meikle3,5,* \\ ${ }^{1}$ Monash Centre for Health Research and Implementation, School of Public Health and Preventative \\ Medicine, Monash University, Clayton, Victoria, Australia \\ 2The Robinson Research Institute, University of Adelaide, North Adelaide, South Australia, Australia \\ ${ }_{3}^{3}$ Metabolomics Laboratory, Baker IDI Heart and Diabetes Institute, Melbourne, Victoria, Australia \\ ${ }^{4}$ Diabetes and Endocrine Unit, Monash Health, Clayton, Victoria, Australia \\ ${ }^{5}$ Department of Biochemistry and Molecular Biology, University of Melbourne, Melbourne, Victoria, Australia \\ *(These authors share joint senior authorship)
}

Correspondence should be addressed to L J Moran Email lisa.moran@monash.edu

\begin{abstract}
Polycystic ovary syndrome (PCOS) affects up to $18 \%$ of reproductive-aged women with reproductive and metabolic complications. While lipidomics can identify associations between lipid species and metabolic diseases, no research has examined the association of lipid species with the pathophysiological features of PCOS. The aim of this study was to examine the lipidomic profile in women with and without PCOS. This study was a cross-sectional study in 156 age-matched pre-menopausal women (18-45 years, $\mathrm{BMI}>20 \mathrm{~kg} / \mathrm{m}^{2} ; n=92$ with PCOS, $n=64$ without PCOS). Outcomes included the association between the plasma lipidomic profile (325 lipid species (24 classes) using liquid chromatography mass spectrometry) and PCOS, adiposity, homeostasis assessment of insulin resistance (HOMA), sex hormone-binding globulin (SHBG) and free androgen index (FAI). There were no associations of the lipidomic profile with PCOS or testosterone. HOMA was positively associated with 2 classes (dihydroceramide and triacylglycerol), SHBG was inversely associated with 2 classes (diacylglycerol and triacylglycerol), FAl was positively associated with 8 classes (ceramide, phosphatidylcholine, lysophosphatidylcholine, phosphatidylethanolamine, lysophosphatidylethanolamine, phosphatidylinositol, diacylglycerol and triacylglycerol) and waist circumference was associated with 8 classes (4 positively (dihydroceramide, phosphatidylglycerol, diacylglycerol and triacylglycerol) and 4 inversely (trihexosylceramide, $\mathrm{G}_{\mathrm{M} 3}$ ganglioside, alkenylphosphatidylcholine and alkylphosphatidylethanolamine)). The lipidomic profile was primarily related to central adiposity and FAI in women with or without PCOS. This supports prior findings that adiposity is a key driver of dyslipidaemia in PCOS and highlights the need for weight management through lifestyle interventions.
\end{abstract}

Key Words
- polycystic ovary syndrome
- lipidomics
- obesity
- hyperandrogenism
- insulin resistance

Journal of Molecular Endocrinology (2017) 59, 93-104

\section{Introduction}

Polycystic ovary syndrome (PCOS) is the most common cause of anovulatory infertility in reproductive-aged women affecting 12-18\% of women (March et al. 2010).
It is a reproductive, metabolic and psychological condition underpinned by genetic abnormalities and exacerbated by obesity. Its key pathophysiological features are 
abnormalities in insulin resistance (IR) (Stepto et al. 2013) (75\% of lean and $95 \%$ of overweight and obese women affected) and hyperandrogenism (80\% affected). IR further worsens hyperandrogenism and drives reproductive and metabolic features in PCOS (Diamanti-Kandarakis \& Dunaif 2012).

Although the diagnosis of PCOS is primarily related to reproductive complications (ESHRE/ASRM 2004), there is increasing recognition of the metabolic consequences of PCOS. However, screening and preventative strategies addressing these sequalae are yet to be optimised. Women with PCOS have a range of traditional and novel risk factors for type 2 diabetes (T2D) and cardiovascular disease (CVD) including hyperlipidaemia, hypertension, inflammation, oxidative stress, procoagulation, impaired fibrinolysis and early vascular markers of atherosclerosis including endothelial dysfunction, arterial stiffness, increased carotid intima media wall thickness, presence of carotid plaque and increased coronary artery calcification (Meyer et al. 2005a,b, Toulis et al. 2011). They also have an increased prevalence of impaired glucose tolerance (IGT) and type 2 diabetes mellitus compared to women without PCOS in systematic reviews and meta-analyses, and they have an apparent increase in cardiovascular disease (Moran et al. 2010, de Groot et al. 2011). This highlights women with PCOS as a population with a greater metabolic risk and a resultant significant health burden.

Dyslipidaemia is very common in women with PCOS, predominantly involving elevated triglycerides and lower high-density lipoproteins (HDL) cholesterol (Cussons et al. 2006). Women with PCOS also have significant qualitative alterations in low-density lipoprotein (LDL) and HDL with increased levels of atherogenic small, dense LDL (subclasses III and IV) (Berneis et al. 2007) and HDL 3c and decreased levels of HDL2a (Legro et al. 1999). Lipidomics enables the characterisation of hundreds of molecular lipid species from biological samples thereby offering the potential for plasma lipid profiling in different disease states. Lipidomics has been previously used for the identification of lipid biomarkers in diseases such as obesity, IR, abnormal glucose tolerance and CVD (Meikle et al. 2013, Bellis et al. 2014, Tonks et al. 2016). Differential lipidomic profiles reported include elevated sphingolipids, diacylglycerol, triacylglycerol, cholesteryl ester and alkylphosphatidylcholine and lower lysophosphatidylcholine and lysoalkylphosphatidylcholine in IR (Tonks et al. 2016) or higher diacylglycerol, triacylglycerol, cholesteryl esters, sphingolipids, phosphatidylethanolamine, phosphatidylglycerol and phosphatidylinositol and negative associations with alkylphosphatidylcholine and alkenylphosphatidylcholine in abnormal glucose tolerance (Meikle et al. 2013).

Given the IR and cardiometabolic risk in PCOS, abnormalities in lipidomic profiling may be expected and could provide insights into defects in lipid metabolism. Only one small study has examined lipidomics in $n=40$ women with PCOS compared to $n=40$ women without PCOS and reported increases in triacyglycerols and sphingomyelinsand decreasesinlysophosphatidylcholines and phosphatidylethanolamines (Haoula et al. 2015). However, this small sample-sized study focused solely on the relationship between the lipidomic profile and PCOS status and did not assess the contribution of the key aetiological factors of hyperinsulinaemia and hyperandrogenism to changes in these lipid classes. The aim of this study was firstly to assess if any differences occur in the lipidomic profile in women with and without PCOS and secondly to assess the associations of the lipidomic with the pathophysiological features of adiposity, insulin resistance or hyperandrogenism underpinning PCOS.

\section{Materials and methods}

\section{Participants}

This secondary analysis is a cross-sectional study of biobanked samples from the baseline measurements of three clinical trials (Meyer et al. 2005b, Moran et al. 2011, Lambert et al. 2015) including women with $(n=92)$ and without $(n=64)$ PCOS. This study subset was selected on the basis of comparable recruitment methods, ages for the women with and without PCOS and all participants being non-smokers and not using blood pressure or lipid lowering medication (including fish oil). Study recruitment and inclusion criteria have been previously described (Meyer et al. 2005b, Moran et al. 2011, Lambert et al. 2015).

The studies comprised pre-menopausal women aged 18-45 years who were overweight or obese (body mass index $(\mathrm{BMI})>25 \mathrm{~kg} / \mathrm{m}^{2}$ ) $($ Meyer et al. 2005b, Moran et al. 2011) or healthy weight, overweight and obese (BMI $20-40 \mathrm{~kg} / \mathrm{m}^{2}$ ) (Lambert et al. 2015). All women had PCOS meeting the European Society for Human Reproduction and Embryology/American Society for Reproductive Medicine (ESHRE/ASRM) diagnosis comprising the presence of two of the three features of hyperandrogenism (either clinical (hirsutism by elevated Ferriman-Gallwey score) or biochemical (elevated testosterone or free

Published by Bioscientifica Ltd. 
androgen index (FAI)), oligo- or amenorrhoea and presence of polycystic ovaries on ultrasound). Of these women, $n=77$ had a diagnosis also consistent with the National Institute of Health (NIH) criteria, while $n=15$ had a diagnosis solely based on the ESHRE/ASRM criteria (non-NIH). Controls in two studies had no history of diagnosed PCOS, and were included on the basis that they did not have more than one PCOS feature (Meyer et al. 2005b, Moran et al. 2011). In the other study, all control women reported regular menstrual cycles, had no history of menstrual dysfunction and demonstrated no evidence of hirsutism and hyperandrogenism based on biochemical analysis of testosterone, sex hormone-binding globulin and free androgen levels (Lambert et al. 2015). Exclusion criteria were type 1 or 2 diabetes mellitus, pregnancy, breastfeeding, secondary hypertension not related to PCOS, cardiovascular, cerebrovascular, liver diseases, severely impaired renal function, severe mental illness and endocrine disorders (congenital adrenal hyperplasia, androgen-secreting tumours, Cushing's syndrome, hyperprolactinaemia, thyroid dysfunction and adrenal disorders). All participants had ceased insulin-sensitising or reproductive hormonal medication for at least 3 months prior to baseline measurements. The studies received ethics approval from Monash University and Monash Health Human Ethics Committees, and all participants gave written informed consent.

\section{Clinical and biochemical measurements}

Following an overnight fast, height and weight were measured and BMI was calculated. Waist circumference (WC) was measured to the nearest $0.5 \mathrm{~cm}$ directly on the skin at the level of midway between the lateral lower rib margin and the iliac crest. Resting systolic blood pressure (SBP) and diastolic blood pressure (DBP) were measured as previously described (Meyer et al. 2005b, Moran et al. 2011, Lambert et al. 2015). Fasting venous blood samples were taken for analysis of glucose, insulin, highly sensitive C-reactive protein (hsCRP), lipids (total cholesterol, HDL cholesterol, LDL cholesterol, triglycerides) as previously described (Meyer et al. 2005b, Moran et al. 2011, Lambert et al. 2015). The homeostasis assessment of insulin resistance (HOMA-IR) was calculated using the following formula: ((fasting insulin) $\times$ (fasting glucose))/22.5. Testosterone was analysed by liquid chromatography mass spectrometry as previously described (Harwood \& Handelsman 2009) but modified to incorporate ultra-pressure liquid chromatography (KeskiRahkonen et al. 2015). The steroid measurements were calibrated against certified reference materials (National Measurement Institute, North Ryde, Australia). The limits of quantification (defined by FDA/EMEA as lowest detectable measurement with $\mathrm{CV}<20 \%$ ) were $0.025 \mathrm{ng} / \mathrm{mL}$ $(0.09 \mathrm{nmol} / \mathrm{L})$. For steroid measurements with values below the limit of quantitation, for data analysis, their concentrations were imputed as 0.5 times the lower limit of quantification for that steroid. Sex hormone-binding globulin (SHBG) was measured by chemiluminescent immunoassay (Immulite 1000, EURO/Diagnostics Products Corp. Ltd., Los Angeles, CA, USA) (Meyer et al. 2005b, Moran et al. 2011) or (Beckman Coulter Unicel DXI 800 (Beckman Coulter, Lane Cove, NSW, Australia) (Lambert et al. 2015)). FAI was calculated using the following formula: ((testosterone $\times$ SHBG)/100). Serum samples were stored at $-80^{\circ} \mathrm{C}$ until analysis.

\section{Lipid extraction and lipidomic analysis}

Lipidomic analysis was performed as previously described (Weir et al. 2013). Plasma samples $(10 \mu \mathrm{L})$ were randomized, and then 20 volumes of $\mathrm{CHCl}_{3}: \mathrm{MeOH}$ (2:1) and $10 \mu \mathrm{L}$ of an internal standard mix containing between 50 and $1000 \mathrm{pmol}$ each of 23 non-physiological and stable isotope labelled lipid standards were added to each sample. Samples were mixed (rotary mixer, $10 \mathrm{~min}$ ), sonicated (water bath, $30 \mathrm{~min}$ ) and then allowed to stand $(20 \mathrm{~min})$ at room temperature. Samples were then centrifuged $(16,000 \boldsymbol{g}, 10 \mathrm{~min})$, and the supernatant was dried under a stream of nitrogen at $40^{\circ} \mathrm{C}$. The extracted lipids were reconstituted in water saturated $\mathrm{BuOH}(50 \mu \mathrm{L})$ and then mixed with $\mathrm{MeOH}(50 \mu \mathrm{L})$ prior to analysis by electrospray ionization tandem mass spectrometry using a Agilent 1200 liquid chromatography system combined with an Applied Biosystems API 4000 Q/TRAP mass spectrometer with a turbo-ionspray source $\left(350^{\circ} \mathrm{C}\right)$ and Analyst 1.5 data system. Multiple Reaction Monitoring (MRM) experiments were used for the major species of each lipid class previously identified in plasma (Weir et al. 2013). Relative lipid amounts were calculated by relating the peak area of each species to the peak area of the corresponding stable isotope or non-physiological internal standard. Total lipid classes (24) were calculated from the sum of the individual lipid species within each class (325 species total) (Weir et al. 2013).

\section{Statistical analysis}

All analyses were performed using MATLAB 2013a (MATHWORKS Inc, MA, USA) by P A M. Data were

Published by Bioscientifica Ltd 
expressed as median and interquartile range unless otherwise stated. Baseline differences between women with and without PCOS in demographic characteristics were assessed using Mann-Whitney tests. Prior to association analysis, data for each lipid class, subclass or species were standardised to the interquartile range (IQR) for ease of interpretation. Principal component analysis (PCA) was performed to provide an unbiased view of lipid profile variation in relation to PCOS status as well as WC, HOMA-IR, SHBG and FAI. The ability of the principal components to stratify the population based on PCOS status WC, HOMA-IR, SHBG and FAI was determined by $t$-tests (PCOS) or Spearman's correlation coefficients (WC, HOMA-IR, SHBG and FAI). Unadjusted binary logistic regression analyses were performed to determine the association of lipid classes, subclasses and individual lipid species with PCOS status. Subsequently, models were adjusted for two sets of covariates: (1) age and WC (2) age, WC, HOMA, FAI, SBP and CRP. For a given lipid measurement, the resultant odds ratio (OR) denotes the number of times a female with a measurement in the 75th percentile is more likely to have PCOS than a female individual with a measurement in the 25th percentile. Linear regression adjusted for PCOS status were used to determine the linear association between lipids and WC, total testosterone, SHBG, FAI or HOMA. The linear regression models were further adjusted for (1) age for WC and (2) age and WC for total testosterone, SHBG, FAI or HOMA. The resultant beta-coefficients denote the change in WC, total testosterone, SHBG, FAI or HOMA with an interquartile range increase in the given lipid measurement. For all analyses, the statistical significance was determined at the level of $<0.05$ after correcting for multiple comparisons as we are measuring hundreds of lipids and looking for their associations simultaneously using the Benjamini-Hochberg method (Benjamini \& Hockberg 1995).

\section{Results}

Baseline characteristics of women with and without PCOS are presented in Table 1 . Women with PCOS had higher weight, BMI, insulin, HOMA, testosterone, FAI, triglycerides, SBP and CRP and lower SHBG than women without PCOS. The principal components PC1, PC2 and PC3 derived from the lipid dataset explained 63.9, 19.1 and $7.6 \%$ of variance. There were no significant differences in PC1, PC2 or PC3 values of women with and without PCOS (Table 2, Supplementary Fig. 1, see section on supplementary data given at the end of this article). However, PC1, PC2 and PC3 were able to stratify WC, HOMA-IR, SHBG and FAI (Table 2, Supplementary Figs 2 and 3). The correlation of WC, HOMA-IR and FAI with PC1, PC2 and PC3 were opposite to the correlation of SHBG with the same components (Table 2).

There were no differences in lipid classes or species between women with and without PCOS either on unadjusted analysis or on adjustment for age and WC or for age, WC, HOMA, FAI, SBP or CRP. There were no lipid classes or species associated with testosterone

Table 1 Baseline characteristics of participants.

\begin{tabular}{l}
\hline Characteristic \\
\hline Age (years) \\
Weight $(\mathrm{kg})$ \\
BMI $\left(\mathrm{kg} / \mathrm{m}^{2}\right)$ \\
Waist circumference $(\mathrm{cm})$ \\
Glucose $(\mathrm{mmol} / \mathrm{L})$ \\
Insulin $(\mu \mathrm{U} / \mathrm{mL})$ \\
HOMA \\
Testosterone (nmol/L) \\
SHBG (nmol/L) \\
FAI \\
Total cholesterol (mmol/L) \\
HDL (mmol/L) \\
LDL (mmol/L) \\
Triglycerides (mmol/L) \\
SBP $(\mathrm{mmHg})$ \\
DBP $(\mathrm{mmHg})$ \\
CRP $(\mathrm{mg} / \mathrm{L})$
\end{tabular}

\begin{tabular}{c}
\hline $\operatorname{PCOS}(N=92)$ \\
\hline $34.8(30.5,38.7)$ \\
$91.3(78.2,101.3)$ \\
$33.9(29.9,38.6)$ \\
$100.7(92.8,111.2)$ \\
$4.6(4.3,4.9)$ \\
$16(9.7,21.0)$ \\
$3.3(2.0,4.5)$ \\
$0.30(0.22,0.42)$ \\
$34.0(23.5,43.3)$ \\
$0.93(0.56,1.40)$ \\
$5.4(4.6,6.1)$ \\
$1.2(1.1,1.4)$ \\
$3.4(2.7,4.0)$ \\
$1.2(0.8,1.8)$ \\
$115.0(110.0,121.2)$ \\
$70.0(65.3,74.3)$ \\
$4.1(2.2,7.9)$
\end{tabular}

\begin{tabular}{ccc}
\hline Non-PCOS $(N=64)$ & & P-value \\
\cline { 1 - 2 } $35.7(30.1,42.3)$ & & 0.608 \\
$84.0(71.9,93.8)$ & & 0.024 \\
$30.0(27.3,35.3)$ & 0.004 \\
$97.0(89.8,108.3)$ & 0.212 \\
$4.6(4.4,4.9)$ & 0.945 \\
$11.5(7.5,17.0)$ & 0.006 \\
$2.3(1.5,3.5)$ & 0.016 \\
$0.20(0.13,0.31)$ & $<0.001$ \\
$44.0(33.7,61.9)$ & $<0.001$ \\
$0.45(0.21,0.83)$ & $<0.001$ \\
$4.9(4.3,5.5)$ & 0.059 \\
$1.2(1.1,1.5)$ & 0.750 \\
$3.2(2.6,3.9)$ & 0.217 \\
$0.90(0.60,1.20)$ & $<0.001$ \\
$110.8(104.5,120.5)$ & 0.001 \\
$67(62.8,74)$ & 0.175 \\
$2(0.6,4)$ & $<0.001$
\end{tabular}

Data are expressed as median (IQR) and were analysed using Mann-Whitney test.

BMI, body mass index; CRP, C-reactive protein; DBP, diastolic blood pressure; FAl, free androgen index; HDL, high-density lipoprotein cholesterol; HOMA, homeostasis assessment of insulin resistance; LDL, low-density lipoprotein cholesterol; SBP, systolic blood pressure; SHBG, sex hormone-binding globulin.

http://jme.endocrinology-journals.org DOI: 10.1530/JME-17-0023
() 2017 Society for Endocrinology Printed in Great Britain
Published by Bioscientifica Ltd 
Table 2 Principal component correlation analysis of plasma lipid species.

\begin{tabular}{|c|c|c|c|c|c|c|}
\hline \multirow[b]{2}{*}{ Characteristica } & \multicolumn{2}{|c|}{ PC1 } & \multicolumn{2}{|c|}{ PC2 } & \multicolumn{2}{|c|}{ PC3 } \\
\hline & Coefficient ${ }^{b}$ & $P$-valuec & Coefficient ${ }^{b}$ & $P$-valuec & Coefficient ${ }^{b}$ & $P$-value \\
\hline PCOS & & 8.97E-02 & & 5.22E-01 & & 2.23E-01 \\
\hline WC & -0.289 & 2.67E-04 & 0.268 & 7.30E-04 & -0.248 & 1.85E-03 \\
\hline HOMA-IR & -0.197 & $1.46 E-02$ & 0.294 & 2.14E-04 & -0.146 & 7.08E-02 \\
\hline SHBG & 0.295 & 1.83E-04 & -0.25 & $1.63 E-03$ & 0.34 & 1.42E-05 \\
\hline FAl & -0.269 & 1.01E-03 & 0.17 & 4.07E-02 & -0.248 & 2.55E-03 \\
\hline
\end{tabular}

aPCOS, polycystic ovary syndrome; WC, waist circumference; SHBG, sex hormone-binding globulin; FAl, free androgen index; bcoefficients based on Spearman's correlation; cP values based on Spearman's correlation between principal components and characteristic values. Values in bold are statistically significant.

(Supplementary Tables 1 and 2). There were no differences in lipid classes or species between women with and without PCOS when subdivided as NIH or non-NIH criteria (data not shown).

WC was associated with 8 lipid classes (4 positively (dihydroceramide, phosphatidylglycerol, diacylglycerol and triacylglycerol) and 4 inversely (trihexosylceramide, $\mathrm{G}_{\mathrm{M} 3}$ ganglioside, alkenylphosphatidylcholine and alkylphosphatidylethanolamine)) in models adjusted for PCOS and age (Table 3). In adjusted models, WC was associated with 123 lipid species. It was positively associated with species of dihydroceramide, ceramide, dihexosylceramide, trihexosylceramide, sphingomyelin, phosphatidylethanolamine, phosphatidylglycerol, phosphatidylinositol, triacylglycerol and inversely associated with species of dihexosylceramide, trihexosylceramide, $\mathrm{G}_{\mathrm{M} 3}$ ganglioside, alkylphosphatidylcholine, alkenylphosphatidylcholine, lysophosphatidylcholine, lysoalkylphosphatidylcholine, alkylphosphatidylethanolamine and alkenylphosphatidylethanolamine. It was also both positively (32:0, 38:2, 40:4) and inversely (18:1-18:3, 18:2-20:4, 16:0-22:6, 39:5, 40:7, 40:8) associated with phosphatidylcholine and positively $(16: 1,18: 1)$ and

Table 3 Association of lipid classes with waist circumference.

\begin{tabular}{l} 
Predictor \\
\hline Dihydroceramide \\
Ceramide \\
Monohexocylceramide \\
Dihexosylceramide \\
Trihexosylceramide \\
$\mathrm{G}_{\text {Mz ganglioside }}$ \\
Sphingomyelin \\
Phosphatidylcholine \\
Alkylphosphatidylcholine \\
Alkenylphosphatidylcholine \\
Lysophosphatidylcholine \\
Lysoalkylphosphatidylcholine \\
Phosphatidylethanolamine \\
Alkylphosphatidylethanolamine \\
Alkenylphosphatidylethanolamine \\
Lysophosphatidylethanolamine \\
Phosphatidylinositol \\
Lysophosphatidylinositol \\
Phosphatidylserine \\
Phosphatidylglycerol \\
Free cholesterol \\
Cholesterol ester \\
Diacylglycerol \\
Triacylglycerol
\end{tabular}

\begin{tabular}{c}
$\beta$-Coefficient $(95 \% \mathrm{Cl})$ \\
Adjusted - Model 1a \\
\hline $\mathbf{6 . 0}(\mathbf{2 . 7}, \mathbf{9 . 2})$ \\
$1.8(-1.2,4.7)$ \\
$-2.8(-5.6,0.03)$ \\
$-2.3(-5.1,0.59)$ \\
$-\mathbf{4 . 4}(-\mathbf{7 . 8}, \mathbf{- 1 . 0})$ \\
$-\mathbf{3 . 4}(-\mathbf{5 . 7}, \mathbf{- 1 . 1 )}$ \\
$1.9(-1.1,4.8)$ \\
$0.47(-2.7,3.6)$ \\
$-3.3(-6.4,-0.24)$ \\
$-\mathbf{5 . 3}(-\mathbf{7 . 7},-\mathbf{3 . 0})$ \\
$-1.2(-3.6,1.3)$ \\
$-1.7(-4.1,0.72)$ \\
$2.8(0.26,5.4)$ \\
$-\mathbf{3 . 6}(-\mathbf{6 . 1},-\mathbf{1 . 1})$ \\
$-2.0(-4.3,0.35)$ \\
$-1.7(-4.3,1.0)$ \\
$1.5(-1.4,4.4)$ \\
$0.26(-2.2,2.7)$ \\
$2.3(-0.003,4.6)$ \\
$\mathbf{5 . 2}(\mathbf{2 . 5}, \mathbf{7 . 9})$ \\
$0.89(-1.8,3.6)$ \\
$-2.4(-4.9,0.05)$ \\
$\mathbf{6 . 2}(\mathbf{3 . 7}, \mathbf{8 . 7})$ \\
$\mathbf{7 . 5}(\mathbf{4 . 4}, \mathbf{1 0 . 6})$ \\
\end{tabular}

\begin{tabular}{r}
\hline P-value \\
\hline $\mathbf{0 . 0 0 2}$ \\
0.302 \\
0.105 \\
0.196 \\
$\mathbf{0 . 0 3 6}$ \\
$\mathbf{0 . 0 1 5}$ \\
0.300 \\
0.805 \\
0.088 \\
$<\mathbf{0 . 0 0 1}$ \\
0.415 \\
0.255 \\
0.086 \\
$\mathbf{0 . 0 1 7}$ \\
0.170 \\
0.300 \\
0.376 \\
0.836 \\
0.105 \\
$\mathbf{0 . 0 0 1}$ \\
0.563 \\
0.105 \\
$<\mathbf{0 . 0 0 1}$ \\
$<\mathbf{0 . 0 0 1}$
\end{tabular}

\begin{tabular}{|c|c|}
\hline $\begin{array}{l}\boldsymbol{\beta} \text {-Coefficient }(95 \% \mathrm{Cl}) \\
\text { Adjusted - Model } \mathbf{2}^{\mathbf{b}}\end{array}$ & P-value \\
\hline $5.9(2.6,9.2)$ & 0.003 \\
\hline $1.6(-1.3,4.6)$ & 0.351 \\
\hline$-2.9(-5.7,-0.05)$ & 0.104 \\
\hline$-2.3(-5.2,0.55)$ & 0.185 \\
\hline$-4.4(-7.8,-1.0)$ & 0.037 \\
\hline$-3.5(-5.7,-1.2)$ & 0.014 \\
\hline $1.8(-1.3,4.8)$ & 0.342 \\
\hline $0.25(-3.0,3.5)$ & 0.892 \\
\hline$-3.3(-6.4,-0.16)$ & 0.098 \\
\hline$-5.3(-7.7,-2.9)$ & $<0.001$ \\
\hline$-1.2(-3.6,1.3)$ & 0.425 \\
\hline$-1.6(-4.1,0.81)$ & 0.287 \\
\hline $2.8(0.18,5.4)$ & 0.098 \\
\hline$-3.7(-6.2,-1.2)$ & 0.015 \\
\hline$-2.0(-4.3,0.36)$ & 0.171 \\
\hline$-1.7(-4.4,1.0)$ & 0.293 \\
\hline $1.4(-1.6,4.3)$ & 0.425 \\
\hline $0.17(-2.3,2.6)$ & 0.892 \\
\hline $2.3(-0.09,4.6)$ & 0.113 \\
\hline $5.2(2.5,8.0)$ & 0.002 \\
\hline $0.72(-2.1,3.5)$ & 0.676 \\
\hline$-2.4(-4.9,0.03)$ & 0.110 \\
\hline $6.3(3.7,8.8)$ & $<0.001$ \\
\hline $7.6(4.4,10.8)$ & $<0.001$ \\
\hline
\end{tabular}

Data were analysed using linear regression and expressed as $\beta$-coefficient ( $95 \%$ confidence interval). $P$ values are corrected for multiple comparisons using the Benjamini-Hochberg method. Values in bold are statistically significant.

aModel 1: PCOS; bModel 2: PCOS, age. 
inversely $(20: 5,22: 6)$ associated with cholesteryl ester (Supplementary Table 3).

SHBG was inversely associated with 2 lipid classes (diacylglycerol and triacylglycerol) in models adjusting for PCOS, age and WC (Table 4). In adjusted models, SHBG was associated with 33 lipid species. Specifically, it was positively associated with alkylphosphatidylcholine and alkenylphosphatidylcholine and inversely associated with diacylglycerol and triacylglycerol (Supplementary Table 4). FAI was positively associated with 8 lipid classes (ceramide, phosphatidylcholine, lysophosphatidylcholine, phosphatidylethanolamine, lysophosphatidylethanolamine, phosphatidylinositol, diacylglycerol and triacylglycerol) in models adjusted for PCOS, age and WC (Table 5). In adjusted models, FAI was associated with 100 lipid species. Specifically, it was positively associated with ceramide, alkylphosphatidylcholine, lysophosphatidylcholine, phosphatidylethanolamine, lysophosphatidylethanolamine, phosphatidylinositol, cholesteryl ester, diacylglycerol and triacylglycerol (Supplementary Table 5). HOMA was positively associated with 2 lipid classes (dihydroceramide and triacylglycerol) in models adjusted for PCOS, age and WC (Table 6). In adjusted models, HOMA was associated with 11 lipid species. Specifically, it was positively associated with ceramide, sphingomyelin, phosphatidylcholine, diacylglycerol and triacylglycerol (Supplementary Table 6).

\section{Discussion}

In the largest study assessing lipidomics in women with PCOS, we report no differences in classes or species between women with or without PCOS and no association of classes or species with total testosterone. We report in both women with and without PCOS that the strongest association of lipid classes and species was with WC with the second strongest association being with FAI as the product of insulin resistance (represented by SHBG) and hyperandrogenism.

We report here no differences in lipid classes or species based on PCOS status. This is in contrast to prior reports of higher triacyglycerols, diacylglycerols and sphingomyelins and lower lysophosphatidylcholines and phosphatidylethanolamines in PCOS compared to controls (Haoula et al. 2015). Although the lipid

Table 4 Association of lipid classes with SHBG.

\begin{tabular}{|c|c|c|c|c|}
\hline & p-Coefficient $(95 \% \mathrm{Cl})$ & & $\boldsymbol{\beta}$-Coefficient $(95 \% \mathrm{Cl})$ & \\
\hline & Adjusted - Model 1a & $P$-value & Adjusted - Model 2b & P-value \\
\hline Dihydroceramide & $-4.1(-8.2,-0.12)$ & 0.120 & $-0.34(-4.2,3.5)$ & 0.969 \\
\hline Ceramide & $-2.6(-6.2,0.99)$ & 0.291 & $-1.4(-4.6,1.8)$ & 0.693 \\
\hline Monohexocylceramide & $0.60(-2.9,4.1)$ & 0.803 & $-0.79(-3.9,2.3)$ & 0.825 \\
\hline Dihexosylceramide & $3.3(-0.10,6.6)$ & 0.142 & $2.3(-0.73,5.3)$ & 0.332 \\
\hline Trihexosylceramide & $5.7(1.8,9.7)$ & 0.017 & $3.2(-0.46,6.9)$ & 0.236 \\
\hline $\mathrm{G}_{\mathrm{M} 3}$ ganglioside & $2.8(-0.17,5.7)$ & 0.146 & $0.77(-1.9,3.4)$ & 0.815 \\
\hline Sphingomyelin & $-1.6(-5.2,1.9)$ & 0.461 & $-0.28(-3.5,3.0)$ & 0.969 \\
\hline Phosphatidylcholine & $-0.56(-4.4,3.3)$ & 0.812 & $-0.16(-3.6,3.3)$ & 0.969 \\
\hline Alkylphosphatidylcholine & $5.6(2.3,8.9)$ & 0.004 & $3.7(0.70,6.7)$ & 0.102 \\
\hline Alkenylphosphatidylcholine & $5.4(2.8,8.1)$ & 0.001 & $2.9(0.30,5.5)$ & 0.145 \\
\hline Lysophosphatidylcholine & $-1.9(4.7,0.97)$ & 0.319 & $-2.4(-4.9,0.089)$ & 0.208 \\
\hline Lysoalkylphosphatidylcholine & $-0.55(-3.5,2.4)$ & 0.803 & $-1.4(-4.0,1.3)$ & 0.628 \\
\hline Phosphatidylethanolamine & $-4.3(-7.3,-1.2)$ & 0.019 & $-2.8(-5.6,-0.042)$ & 0.194 \\
\hline Alkylphosphatidylethanolamine & $2.0(-1.1,5.1)$ & 0.321 & $-0.45(-3.3,2.4)$ & 0.954 \\
\hline Alkenylphosphatidylethanolamine & $1.6(-1.1,4.3)$ & 0.357 & $0.037(-2.4,2.5)$ & 0.977 \\
\hline Lysophosphatidylethanolamine & $-0.4(-3.5,2.8)$ & 0.824 & $-1.5(-4.2,1.3)$ & 0.628 \\
\hline Phosphatidylinositol & $-2.4(-5.9,1.1)$ & 0.306 & $-1.3(-4.5,1.8)$ & 0.693 \\
\hline Lysophosphatidylinositol & $-1.5(-4.5,1.5)$ & 0.436 & $-1.1(-3.7,1.6)$ & 0.693 \\
\hline Phosphatidylserine & $-4.6(-7.3,-1.9)$ & 0.004 & $-3.3(-5.8,-0.83)$ & 0.079 \\
\hline Phosphatidylglycerol & $-6.0(-9.3,-2.7)$ & 0.003 & $-2.8(-6.0,0.38)$ & 0.236 \\
\hline Free cholesterol & $-0.82(-4.1,2.4)$ & 0.744 & $-0.16(-3.1,2.8)$ & 0.969 \\
\hline Cholesteryl ester & $2.4(-0.70,5.4)$ & 0.267 & $0.81(-1.9,3.6)$ & 0.815 \\
\hline Diacylglycerol & $-9.4(-12.3,-6.4)$ & $<0.001$ & $-6.5(-9.6,-3.4)$ & 0.001 \\
\hline Triacylglycerol & $-10.2(-14.0,-6.4)$ & $<0.001$ & $-6.6(-10.5,-2.8)$ & 0.012 \\
\hline
\end{tabular}

Data were analysed using linear regression and expressed as $\beta$-coefficient ( $95 \%$ confidence interval). $P$ values are corrected for multiple comparisons using the Benjamini-Hochberg method. Values in bold are statistically significant.

aModel 1: PCOS; bModel 2: PCOS, age, waist circumference. 
Table 5 Association of lipid classes with FAI.

\begin{tabular}{l}
\hline \\
\hline Dihydroceramide \\
Ceramide \\
Monohexocylceramide \\
Dihexosylceramide \\
Trihexosylceramide \\
GM ganglioside \\
Sphingomyelin \\
Phosphatidylcholine \\
Alkylphosphatidylcholine \\
Alkenylphosphatidylcholine \\
Lysophosphatidylcholine \\
Lysoalkylphosphatidylcholine \\
Phosphatidylethanolamine \\
Alkylphosphatidylethanolamine \\
Alkenylphosphatidylethanolamine \\
Lysophosphatidylethanolamine \\
Phosphatidylinositol \\
Lysophosphatidylinositol \\
Phosphatidylserine \\
Phosphatidylglycerol \\
Free cholesterol \\
Cholesterol ester \\
Diacylglycerol \\
Triacylglycerol
\end{tabular}

\begin{tabular}{|c|}
\hline $\begin{array}{l}\beta \text {-Coefficient }(95 \% \mathrm{Cl}) \\
\text { Adjusted - Model 1a }\end{array}$ \\
\hline $0.11(-0.041,0.26)$ \\
\hline $0.18(0.047,0.31)$ \\
\hline $0.022(-0.11,0.15)$ \\
\hline$-0.082(-0.21,0.049)$ \\
\hline$-0.099(-0.26,0.059)$ \\
\hline$-0.017(-0.13,0.092)$ \\
\hline $0.031(-0.011,0.17)$ \\
\hline $0.20(0.057,0.34)$ \\
\hline$-0.095(-0.24,0.054)$ \\
\hline$-0.087(-0.20,0.027)$ \\
\hline $0.15(0.037,0.26)$ \\
\hline$-0.044(-0.16,0.068)$ \\
\hline $0.20(0.089,0.32)$ \\
\hline$-0.010(-0.13,0.11)$ \\
\hline$-0.014(-0.12,0.093)$ \\
\hline $0.20(0.081,0.32)$ \\
\hline $0.17(0.042,0.31)$ \\
\hline $0.0083(-0.11,0.12)$ \\
\hline $0.12(0.018,0.23)$ \\
\hline $0.20(0.072,0.33)$ \\
\hline $0.025(-0.098,0.15)$ \\
\hline$-0.051(-0.017,0.064)$ \\
\hline $0.33(0.22,0.44)$ \\
\hline $0.37(0.23,0.52)$ \\
\hline
\end{tabular}

\begin{tabular}{c}
\hline P-value \\
\hline 0.306 \\
0.028 \\
0.866 \\
0.357 \\
0.357 \\
0.866 \\
0.866 \\
$\mathbf{0 . 0 2 8}$ \\
0.357 \\
0.299 \\
$\mathbf{0 . 0 2 8}$ \\
0.626 \\
$\mathbf{0 . 0 0 5}$ \\
0.887 \\
0.872 \\
$\mathbf{0 . 0 0 8}$ \\
$\mathbf{0 . 0 2 8}$ \\
0.887 \\
0.056 \\
$\mathbf{0 . 0 1 3}$ \\
0.866 \\
0.577 \\
$<\mathbf{0 . 0 0 1}$ \\
$<\mathbf{0 . 0 0 1}$ \\
\hline
\end{tabular}

\begin{tabular}{c}
$\beta$-Coefficient $(95 \% \mathrm{Cl})$ \\
Adjusted - Model 2 $\mathbf{b}^{\mathbf{b}}$ \\
\hline $0.011(-0.14,0.16)$ \\
$0.16(0.037,0.28)$ \\
$0.066(-0.057,0.19)$ \\
$-0.040(-0.16,0.083)$ \\
$-0.027(-0.18,0.12)$ \\
$0.047(-0.056,0.15)$ \\
$0.0055(-0.12,0.13)$ \\
$\mathbf{0 . 2 2}(\mathbf{0 . 0 8 9}, \mathbf{0 . 3 6})$ \\
$-0.055(-0.19,0.084)$ \\
$-0.0071(-0.12,0.11)$ \\
$\mathbf{0 . 1 6}(\mathbf{0 . 0 6 3 , 0 . 2 7 )}$ \\
$-0.027(-0.13,0.078)$ \\
$\mathbf{0 . 1 7}(\mathbf{0 . 0 6 0}, \mathbf{0 . 2 8})$ \\
$0.068(-0.046,0.18)$ \\
$0.019(-0.08,0.12)$ \\
$\mathbf{0 . 2 4}(\mathbf{0 . 1 3}, \mathbf{0 . 3 5})$ \\
$\mathbf{0 . 1 7}(\mathbf{0 . 0 4 8 , 0 . 2 9 )}$ \\
$0.0024(-0.10,0.11)$ \\
$0.091(0.19,0.19)$ \\
$0.12(-0.0033,0.25)$ \\
$0.027(-0.092,0.15)$ \\
$-0.0072(-0.11,0.10)$ \\
$\mathbf{0 . 2 7}(\mathbf{0 . 1 5}, \mathbf{0 . 3 9})$ \\
$\mathbf{0 . 3 0}(\mathbf{0 . 1 5}, \mathbf{0 . 4 5})$ \\
\end{tabular}

\begin{tabular}{c}
\hline P-value \\
\hline 0.965 \\
0.035 \\
0.591 \\
0.837 \\
0.917 \\
0.688 \\
0.965 \\
$\mathbf{0 . 0 0 9}$ \\
0.752 \\
0.965 \\
$\mathbf{0 . 0 0 9}$ \\
0.917 \\
$\mathbf{0 . 0 1 1}$ \\
0.532 \\
0.917 \\
$\mathbf{0 . 0 0 1}$ \\
$\mathbf{0 . 0 2 6}$ \\
0.965 \\
0.189 \\
0.156 \\
0.917 \\
0.965 \\
$<\mathbf{0 . 0 0 1}$ \\
$\mathbf{0 . 0 0 1}$
\end{tabular}

Data were analysed using linear regression and expressed as $\beta$-coefficient ( $95 \%$ confidence interval). $P$ values are corrected for multiple comparisons using the Benjamini-Hochberg method. Values in bold are statistically significant.

aModel 1: PCOS; bModel 2: PCOS, age, waist circumference.

extraction and analysis was different between studies, this would not be expected to lead to such differences as both analytical procedures have been validated, and the comparison (PCOS vs control) within each study is only by a single method thereby highlighting differences within that PCOS/control group. The differences between PCOS and control groups in the earlier study may relate to the significant difference in BMI between the PCOS and control groups as no adjustment was made for this. Previous studies have reported diacyl and triacylglycerols as well as sphingomyelin to have a positive association with BMI and lysophosphatidylcholine to have a negative association with BMI (Weir et al. 2013). The women with PCOS in this earlier study were also a clinical recruited population and are therefore likely to have a higher BMI (Ezeh et al. 2013) and a more severe reproductive and metabolic presentation in contrast to our community recruited population. This is likely to result in greater differences in lipidomic profile than in our current study. This is in keeping with the women with and without PCOS in this current study having differences in the traditional lipid profile only for triglycerides and that the principle components from our PCA were able to stratify on WC, HOMA-IR, SHBG and FAI but not PCOS. Here, this demonstrates that unlike PCOS, these characteristics were significantly related to the overall lipidomic profile in these women. Our findings are consistent with much of the PCOS literature suggesting that while IR is inherent in the condition, lipid abnormalities are primarily weight related in PCOS (Wild et al. 2011) and highlights the need for proper adjustment in the analysis of data in these patients. Further research should examine lipidomic differences across the range of heterogeneity of PCOS including both lean and overweight women, women with differing degrees of IR, dyslipidaemia and glucose intolerance and women with different diagnostic phenotypes.

We observed here positive associations of IR with dihydroceramide and triacylglycerol classes and specific diacylglycerol, sphingomyelin and phosphatidylcholine species. These associations occurred independent of PCOS, age and central adiposity. This is consistent with prior reports of elevated plasma or muscle diacylglycerol, triacylglycerol and sphingolipids for those women with IR compared to those without IR (Tonks et al. 2016). It is also consistent with the elevation of the specific phosphatidylcholine species observed here (phosphatidylcholine 38:3) in individuals with metabolic

Published by Bioscientifica Ltd. 
Table 6 Association of lipid classes with HOMA.

\begin{tabular}{|c|c|c|c|c|}
\hline & $\begin{array}{l}\text { } \beta \text {-Coefficient }(95 \% \mathrm{Cl}) \\
\text { Adjusted - Model 1a }\end{array}$ & P-value & $\begin{array}{l}\boldsymbol{\beta} \text {-Coefficient }(95 \% \mathrm{Cl}) \\
\text { Adjusted - Model } \mathbf{2}^{\mathbf{b}}\end{array}$ & $P$-value \\
\hline Dihydroceramide & $1.007(0.544,1.469)$ & 0.001 & $0.888(0.411,1.364)$ & 0.009 \\
\hline Ceramide & $0.254(-0.184,0.692)$ & 0.387 & $0.257(-0.167,0.680)$ & 0.379 \\
\hline Monohexocylceramide & $0.196(-0.225,0.617)$ & 0.459 & $0.400(-0.008,0.808)$ & 0.136 \\
\hline Dihexosylceramide & $0.261(-0.164,0.686)$ & 0.368 & $0.374(-0.031,0.779)$ & 0.158 \\
\hline Trihexosylceramide & $-0.076(-0.576,0.424)$ & 0.799 & $0.166(-0.327,0.658)$ & 0.645 \\
\hline $\mathrm{G}_{\mathrm{M} 3}$ ganglioside & $0.162(-0.203,0.527)$ & 0.462 & $0.372(0.021,0.722)$ & 0.118 \\
\hline Sphingomyelin & $0.473(0.038,0.907)$ & 0.139 & $0.527(0.095,0.958)$ & 0.086 \\
\hline Phosphatidylcholine & $0.453(-0.012,0.918)$ & 0.200 & $0.559(0.111,1.006)$ & 0.086 \\
\hline Alkylphosphatidylcholine & $-0.020(-0.432,0.391)$ & 0.922 & $0.159(-0.246,0.564)$ & 0.590 \\
\hline Alkenylphosphatidylcholine & $-0.278(-0.611,0.055)$ & 0.237 & $-0.039(-0.386,0.308)$ & 0.902 \\
\hline Lysophosphatidylcholine & $-0.233(-0.581,0.155)$ & 0.354 & $-0.171(-0.507,0.165)$ & 0.452 \\
\hline Lysoalkylphosphatidylcholine & $-0.232(-0.590,0.126)$ & 0.354 & $-0.185(-0.532,0.161)$ & 0.445 \\
\hline Phosphatidylethanolamine & $0.336(-0.044,0.716)$ & 0.237 & $0.274(-0.097,0.646)$ & 0.278 \\
\hline Alkylphosphatidylethanolamine & $-0.206(-0.582,0.171)$ & 0.403 & $-0.022(-0.394,0.349)$ & 0.946 \\
\hline Alkenylphosphatidylethanolamine & $-0.073(-0.409,0.263)$ & 0.733 & $0.051(-0.278,0.379)$ & 0.885 \\
\hline Lysophosphatidylethanolamine & $-0.101(-0.484,0.282)$ & 0.693 & $0.011(-0.361,0.382)$ & 0.956 \\
\hline Phosphatidylinositol & $0.277(-0.151,0.704)$ & 0.354 & $0.288(-0.129,0.706)$ & 0.305 \\
\hline Lysophosphatidylinositol & $0.303(-0.065,0.671)$ & 0.237 & $0.348(-0.002,0.698)$ & 0.136 \\
\hline Phosphatidylserine & $0.403(0.061,0.744)$ & 0.128 & $0.367(0.032,0.703)$ & 0.115 \\
\hline Phosphatidylglycerol & $0.471(0.059,0.883)$ & 0.128 & $0.315(-0.108,0.738)$ & 0.278 \\
\hline Free cholesterol & $0.337(-0.055,0.728)$ & 0.237 & $0.431(0.043,0.819)$ & 0.115 \\
\hline Cholesterol ester & $-0.177(-0.545,0.193)$ & 0.459 & $-0.053(-0.413,0.307)$ & 0.885 \\
\hline Diacylglycerol & $0.700(0.316,1.084)$ & 0.004 & $0.566(0.151,0.981)$ & 0.067 \\
\hline Triacylglycerol & $0.935(0.454,1.416)$ & 0.002 & $0.810(0.296,1.323)$ & 0.029 \\
\hline
\end{tabular}

Data were analysed using linear regression and expressed as $\beta$-coefficient ( $95 \%$ confidence interval). $P$ values are corrected for multiple comparisons using the Benjamini-Hochberg method. Values in bold are statistically significant.

aModel 1: PCOS; bModel 2: PCOS, age, waist circumference.

syndrome (Kulkarni et al. 2013). However, our findings did not align with all previous associations with IR such as higher cholesterol ester or lower lysophosphatidylcholine or lysoalkylphosphatidylcholine (Tonks et al. 2016). This may be due to the use of HOMA as a surrogate marker of IR here compared to the gold-standard measure of the euglycaemic hyperinsulinaemic clamp (Tonks et al. 2016). The lipid species and class associations observed with HOMA have also been reported with other clinical conditions such as obesity, hypertension, type 2 diabetes and metabolic syndrome. This highlights the relevance of these aberrations with both IR and clinical conditions where IR is a strong aetiological factor (Yang et al. 2016). This supports the contribution of IR to metabolic dysfunction in PCOS (Lee da et al. 2014).

We report here for the first time in either women with or without PCOS a negative association of SHBG with diacylglycerol and triacylglycerol classes and a positive association with alkylphosphatidylcholine and alkenylphosphatidylcholine species. SHBG is inversely related to IR via insulin-reducing hepatic SHBG production in in vitro studies (Plymate et al. 1988), and associations of cardiovascular risk factors with SHBG may be reflective of SHBG as a potential surrogate marker of insulin resistance.
However, lower SHBG was previously associated with a more atherogenic lipid profile consisting of smaller LDL, lower medium LDL and higher triglycerides, VLDL and IDL assessed using ion mobility spectrometry in $n=42$ South Asian Indian women (Kim et al. 2015) with these findings persisting after adjustment for visceral adiposity and surrogate markers of IR. In keeping with this, in the general population, SHBG is associated with DM2 independent of surrogate measures of IR (Haffner et al. 1993, Kalyani et al. 2009), and SHBG, but not testosterone, is associated with the prevalence or incidence of subclinical atherosclerosis (Calderon-Margalit et al. 2010) in women independent of factors including age, BMI, insulin or clinical lipid measurements. This highlights the possibility of either a direct relationship or an as-yet unknown mediator between SHBG and an improved atherogenic lipid profile and supports prior research highlighting that SHBG is a marker of metabolic risk independent of androgen status (Moran et al. 2013).

Here, we found positive associations of FAI with ceramide, cholesterol esters, lysophosphatidylcholine, lysoalkylphosphatidylcholine, lysophosphatidylethanolamine, diacylglycerols and triacylglycerols for both women with and without PCOS.

Published by Bioscientifica Ltd 
A number of these lipidomic differences also occurred independent of IR in this current study. This raises the greater potential relevance of bioavailable testosterone as a potential contributor to cardiometabolic risk in PCOS. This is supported by estimates of free but not total testosterone being associated with the metabolic syndrome in PCOS (Brand et al. 2011). As FAI also incorporates SHBG as a potential marker of IR, this supports FAIs potential utility in capturing both contribution of hyperandrogenism and hyperinsulinaemia as the two key pathophysiological features of PCOS. This is in keeping with overlapping but distinct profiles of lipid species and classes that show opposing associations with FAI compared to SHBG and HOMA. Specifically, FAI was associated with lysophosphatidylcholine, phosphatidylethanolamine, lysophosphatidylethanolamine, cholesterol ester and phosphatidylinositol in addition to species also associated with SHBG and HOMA such as ceramide, alkylphosphatidylcholine, diacylglycerol or triacylglycerols.

However, overall, we reported that the factor most strongly associated with the lipidomic profile here for both women with or without PCOS was WC, which was positively associated with dihydroceramide, phosphatidylglycerol, diacylglycerol and triacylglycerol and inversely associated with trihexosylceramide, $\mathrm{G}_{\mathrm{M} 3}$ ganglioside, alkenylphosphatidylcholine and alkylphosphatidylethanolamine. This profile is largely consistent with previous studies in DM2 or in individuals with obesity or elevated liver or epicardial fat (Kolak et al. 2007, Meikle et al. 2013, Scherer et al. 2015). This highlights that lipid abnormalities in PCOS are likely largely related to adiposity and its distribution in PCOS (Lim et al. 2013). This may be related to factors including elevated hepatic fat (Jones et al. 2012) or IR (Dunaif et al. 1989, Stepto et al. 2013). It is also of interest that despite significant associations of the lipidomic profile with the key pathophysiological features in PCOS of adiposity, reproductive hormones and insulin resistance that overall the higher adiposity, hyperandrogenism and insulin resistance and lower SHBG did not translate to overall differences in the lipidomic profile in PCOS. While this may be related to issues such as the heterogeneity of this study population as previously discussed, this highlights the implications of this work with regard to a broader understanding of the contributing factors to metabolic dysfunction in a wider population of women.

The mechanistic relationship of these lipid classes and species with adiposity, IR, reproductive hormones and metabolic dysfunction and their clinical relevance is still being clarified. Diacylglycerol is associated with IR in animal models through mechanisms including phosphorylation of insulin receptor substrates and inhibition of phosphatidylinositol 3-kinase (Chavez \& Summers 2010). The sphingolipid class comprises lipid species including ceramide, sphingomyelin and dihydroceramide. These are also associated with IR through mechanisms including the upregulation of ceramide synthesis or sphingomyelin breakdown in an inflammatory state (Holland et al. 2011) or the regulation of muscle glucose uptake or hepatic glucose output (Chavez \& Summers 2010). The inverse association of WC with dihexosylceramide may further indicate downregulation of the glycosyltransferases responsible for the conversion of ceramide to dihexosylceramide and trihexosylceramide. The different phosphatidylcholine species may have beneficial metabolic effects including suppression of lipogenesis and enhancement of lipolysis (Shirouchi et al. 2007). Elevated lysophosphatidylcholine may indicate an increase in phospholipase activity which is highly expressed in the atherogenic plaque and associated with atherosclerosis progression (Schmitz \& Ruebsaamen 2010). Phosphatidylglycerol is a metabolic precursor to cardiolipin which is depleted in diabetic myocardium with cardiolipin remodelling occurring very early in the diabetic process (Han et al. 2007). The inverse association of WC with alkenylphosphatidylcholine or alkylphosphatidylethanalomine or the positive association of SHBG with alkylphosphatidylcholine and alkenylphosphatidylcholine is consistent with their susceptibility to oxidative stress and potential role as antioxidants (Wallner \& Schmitz 2011). Lower levels of these lipids in DM2, central adiposity or insulin resistance (as represented by the surrogate marker SHBG) have been previously postulated to be related to decreased biosynthesis or depletion in relation to increased oxidative stress in association with elevated triacylglycerol or diacylglycerol levels (Wallner \& Schmitz 2011).

A strength of this study is the relatively larger sample size than prior publications assessing the lipidomic profile in PCOS (Haoula et al. 2015) that have allowed us to analyse the relationship between plasma lipid species and classes with PCOS while adjusting for other characteristics also known to associate with plasma lipids. We have also been able to extend this association of lipid classes and species with the pathophysiological features of hyperinsulinaemia and hyperandrogenism. We assessed a well-characterised cohort with exclusion of smoking, hormonal contraceptives or use of blood pressure or lipid lowering medication. However, we lacked information on

Published by Bioscientifica Ltd. 
participant characteristics that may also contribute to the lipidomic profile such as diet, physical activity, sedentary behaviour and alcohol consumption (Rauschert et al. 2016). We also note heterogeneity in both women with and without PCOS based on the presence of differing numbers of features of the PCOS diagnostic criteria. We conducted separate analyses based on whether the women with PCOS were diagnosed according to the NIH criteria or not and reported no differences in comparison to our combined analyses. However, we note the small sample size here for the non-NIH PCOS $(n=15)$, which limits the power of these analyses. Future work examining differences in the lipidomic profile in women with PCOS with further consideration of the full range of diagnostic phenotypes, differing degrees of metabolic dysfunction and relevant lifestyle characteristics in adequately powered studies is therefore warranted. This would allow assessment of the relative effects of PCOS diagnosis and modifiable factors of the lipidomic profile and cardiometabolic risk.

While we observed no differences in the lipidomic profile between women with and without PCOS, we report here for the first time that alterations in the lipidomic profile for both women with or without PCOS combined are primarily related to central adiposity. We also report a modest independent association of SHBG, and surrogate markers of insulin resistance with distinct lipidomic classes and species in women with and without PCOS. This provides insight into the relationship between disease pathophysiology and specific alterations in lipoprotein composition and function in women with and without PCOS. This supports prior research suggesting that adiposity is a key driver of dyslipidaemia in PCOS (Lim et al. 2013) and highlights the need for prevention of weight gain through lifestyle intervention.

\section{Supplementary data}

This is linked to the online version of the paper at http://dx.doi.org/10.1530/ JME-17-0023.

\section{Declaration of interest}

All authors declare no conflict of interest that could be perceived as prejudicing the impartiality of the research reported.

\section{Funding}

L $M$ is supported by a South Australian Cardiovascular Research Development Program Fellowship; a program collaboratively funded by the National Heart Foundation, the South Australian Department of Health and the South Australian Health and Medical Research Institute. H T is supported by a fellowship from the National Health and Medical Research Council. This work was supported by the Endocrine Society of Australia Ken Wynne Post-doctoral research award.

\section{Author's contribution statement}

Lisa Moran, Helena Teede and Peter Meikle had substantial contributions to conception and design, or acquisition of data. Lisa Moran, Piyush Munda, Helena Teede and Peter Meike contributed to analysis of data. Lisa Moran, Piyush Munda, Helena Teede and Peter Meike contributed to interpretation of data. Lisa Moran, Piyush Munda, Helena Teede and Peter Meike contributed to drafting the article or revising it critically for important intellectual content. Lisa Moran, Piyush Munda, Helena Teede and Peter Meike had final approval of the version to be published.

\section{Acknowledgements}

We acknowledge Gavin Lambert, Soulmaz Shorakae and Melanie GibsonHelm for assistance with the clinical trials. H J Teede and P J Meikle share joint senior authorship.

\section{References}

Bellis C, Kulkarni H, Mamtani M, Kent JW Jr, Wong G, Weir JM, Barlow CK, Diego V, Almeida M, Dyer TD, et al. 2014 Human plasma lipidome is pleiotropically associated with cardiovascular risk factors and death. Circulation: Cardiovascular Genetics 7 854-863. (doi:10.1161/CIRCGENETICS.114.000600)

Benjamini Y \& Hockberg Y 1995 Controlling the false discovery rate: a practical and powerful approach to multiple testing. Journal of the Royal Statistical Society: Series B (Statistical Methodology) $\mathbf{5 7}$ 289-300.

Berneis K, Rizzo M, Lazzarini V, Fruzzetti F \& Carmina E 2007 Atherogenic lipoprotein phenotype and low-density lipoproteins size and subclasses in women with polycystic ovary syndrome. Journal of Clinical Endocrinology and Metabolism 92 186-189. (doi:10.1210/ jc.2006-1705)

Brand JS, van de Tweel I, Grobbee DE, Emmelot-Vonk MH \& van de Schouw YT 2011 Testosterone, sex hormone-binding globulin and the metabolic syndrome: a systematic review and meta-analysis of observational studies. International Journal of Epidemiology $\mathbf{4 0}$ 189-207. (doi:10.1093/ije/dyq158)

Calderon-Margalit R, Schwartz SM, Wellons MF, Lewis CE, Daviglus ML Schreiner PJ, Williams OD, Sternfeld B, Carr JJ, O'Leary DH, et al. 2010 Prospective association of serum androgens and sex hormonebinding globulin with subclinical cardiovascular disease in young adult women: the 'Coronary Artery Risk Development in Young Adults' women's study. Journal of Clinical Endocrinology and Metabolism 95 4424-4431. (doi:10.1210/jc.2009-2643)

Chavez JA \& Summers SA 2010 Lipid oversupply, selective insulin resistance, and lipotoxicity: molecular mechanisms. Biochimica et Biophysica Acta 1801 252-265. (doi:10.1016/j.bbalip.2009.09.015)

Cussons AJ, Stuckey BG \& Watts GF 2006 Cardiovascular disease in the polycystic ovary syndrome: new insights and perspectives. Atherosclerosis 185 227-239. (doi:10.1016/j. atherosclerosis.2005.10.007)

de Groot PC, Dekkers OM, Romijn JA, Dieben SW \& Helmerhorst FM 2011 PCOS, coronary heart disease, stroke and the influence of obesity: a systematic review and meta-analysis. Human Reproduction Update 17 495-500. (doi:10.1093/humupd/dmr001)

Diamanti-Kandarakis E \& Dunaif A 2012 Insulin resistance and the polycystic ovary syndrome revisited: an update on mechanisms and implications. Endocrine Reviews 33 981-1030. (doi:10.1210/er.20111034)

Dunaif A, Segal KR, Futterweit W \& Dobrjansky A 1989 Profound peripheral insulin resistance, independent of obesity, in polycystic ovary syndrome. Diabetes 38 1165-1174. (doi:10.2337/ diab.38.9.1165) 
ESHRE/ASRM 2004 Revised 2003 consensus on diagnostic criteria and long-term health risks related to polycystic ovary syndrome (PCOS). Human Reproduction 19 41-47.

Ezeh U, Yildiz BO \& Azziz R 2013 Referral bias in defining the phenotype and prevalence of obesity in polycystic ovary syndrome. Journal of Clinical Endocrinology and Metabolism 98 E1088-E1096. (doi:10.1210/jc.2013-1295)

Haffner SM, Valdez RA, Morales PA, Hazuda HP \& Stern MP 1993 Decreased sex hormone-binding globulin predicts noninsulindependent diabetes mellitus in women but not in men. Journal of Clinical Endocrinology and Metabolism 77 56-60.

Han X, Yang J, Yang K, Zhao Z, Abendschein DR \& Gross RW 2007 Alterations in myocardial cardiolipin content and composition occur at the very earliest stages of diabetes: a shotgun lipidomics study. Biochemistry 46 6417-6428. (doi:10.1021/bi7004015)

Haoula Z, Ravipati S, Stekel DJ, Ortori CA, Hodgman C, Daykin C, Raine-Fenning N, Barrett DA \& Atiomo W 2015 Lipidomic analysis of plasma samples from women with polycystic ovary syndrome. Metabolomics 11 657-666. (doi:10.1007/s11306-014-0726-y)

Harwood DT \& Handelsman DJ 2009 Development and validation of a sensitive liquid chromatography-tandem mass spectrometry assay to simultaneously measure androgens and estrogens in serum without derivatization. Clinica Chimica Acta 409 78-84. (doi:10.1016/j. cca.2009.09.003)

Holland WL, Bikman BT, Wang LP, Yuguang G, Sargent KM, Bulchand S, Knotts TA, Shui G, Clegg DJ, Wenk MR, et al. 2011 Lipid-induced insulin resistance mediated by the proinflammatory receptor TLR4 requires saturated fatty acid-induced ceramide biosynthesis in mice. Journal of Clinical Investigation 121 1858-1870. (doi:10.1172/ JCI43378)

Jones H, Sprung VS, Pugh CJ, Daousi C, Irwin A, Aziz N, Adams VL, Thomas EL, Bell JD, Kemp GJ, et al. 2012 Polycystic ovary syndrome with hyperandrogenism is characterized by an increased risk of hepatic steatosis compared to nonhyperandrogenic PCOS phenotypes and healthy controls, independent of obesity and insulin resistance. Journal of Clinical Endocrinology and Metabolism 97 3709-3716. (doi:10.1210/jc.2012-1382)

Kalyani RR, Franco M, Dobs AS, Ouyang P, Vaidya D, Bertoni A, Gapstur SM \& Golden SH 2009 The association of endogenous sex hormones, adiposity, and insulin resistance with incident diabetes in postmenopausal women. Journal of Clinical Endocrinology and Metabolism 94 4127-4135. (doi:10.1210/jc.2009-0910)

Keski-Rahkonen P, Desai R, Jimenez M, Harwood DT \& Handelsman DJ 2015 Measurement of estradiol in human serum by LC-MS/MS using a novel estrogen-specific derivatization reagent. Analytical Chemistry 87 7180-7186. (doi:10.1021/acs.analchem.5b01042)

Kim C, Kong S, Krauss RM, Stanczyk FZ, Reddy ST, Needham BL \& Kanaya AM 2015 Endogenous sex steroid hormones, lipid subfractions, and ectopic adiposity in Asian Indians. Metabolic Syndrome and Related Disorders 13 445-452. (doi:10.1089/ met.2015.0063)

Kolak M, Westerbacka J, Velagapudi VR, Wagsater D, Yetukuri L, Makkonen J, Rissanen A, Hakkinen AM, Lindell M, Bergholm $\mathrm{R}$, et al. 2007 Adipose tissue inflammation and increased ceramide content characterize subjects with high liver fat content independent of obesity. Diabetes 56 1960-1968. (doi:10.2337/db070111)

Kulkarni H, Meikle PJ, Mamtani M, Weir JM, Barlow CK, Jowett JB, Bellis C, Dyer TD, Johnson MP, Rainwater DL, et al. 2013 Variability in associations of phosphatidylcholine molecular species with metabolic syndrome in Mexican-American families. Lipids $\mathbf{4 8}$ 497-503. (doi:10.1007/s11745-013-3781-7)

Lambert EA, Teede H, Sari CI, Jona E, Shorakae S, Woodington K, Hemmes R, Eikelis N, Straznicky NE, De Courten B, et al. 2015 Sympathetic activation and endothelial dysfunction in polycystic ovary syndrome are not explained by either obesity or insulin resistance. Clinical Endocrinology 83 812-819. (doi:10.1111/ cen.12803)

Lee da E, Park SY, Park SY, Lee SR, Chung HW \& Jeong K 2014 Clinical and biochemical profiles according to homeostasis model assessment-insulin resistance (HOMA-IR) in Korean women with polycystic ovary syndrome. Journal of Menopausal Medicine 20 104-110. (doi:10.6118/jmm.2014.20.3.104)

Legro RS, Blanche P, Krauss RM \& Lobo RA 1999 Alterations in lowdensity lipoprotein and high-density lipoprotein subclasses among Hispanic women with polycystic ovary syndrome: influence of insulin and genetic factors. Fertility and Sterility 72 990-995. (doi:10.1016/S0015-0282(99)00401-X)

Lim SS, Norman RJ, Davies MJ \& Moran LJ 2013 The effect of obesity on polycystic ovary syndrome: a systematic review and metaanalysis. Obesity Reviews 14 95-109. (doi:10.1111/j.1467-789X.2012.01053.x)

March WA, Moore VM, Willson KJ, Phillips DI, Norman RJ \& Davies MJ 2010 The prevalence of polycystic ovary syndrome in a community sample assessed under contrasting diagnostic criteria. Human Reproduction 25 544-551. (doi:10.1093/humrep/dep399)

Meikle PJ, Wong G, Barlow CK, Weir JM, Greeve MA, MacIntosh GL, Almasy L, Comuzzie AG, Mahaney MC, Kowalczyk A, et al. 2013 Plasma lipid profiling shows similar associations with prediabetes and type 2 diabetes. PLOS ONE $\mathbf{8}$ e74341. (doi:10.1371/journal. pone.0074341)

Meyer C, McGrath BP, Cameron J, Kotsopoulos D \& Teede HJ 2005a Vascular dysfunction and metabolic parameters in polycystic ovary syndrome. Journal of Clinical Endocrinology and Metabolism 90 4630-4635. (doi:10.1210/jc.2004-1487)

Meyer C, McGrath BP \& Teede HJ 2005b Overweight women with polycystic ovary syndrome have evidence of subclinical cardiovascular disease. Journal of Clinical Endocrinology and Metabolism 90 5711-5716. (doi:10.1210/jc.2005-0011)

Moran LJ, Misso ML, Wild RA \& Norman RJ 2010 Impaired glucose tolerance, type 2 diabetes and metabolic syndrome in polycystic ovary syndrome: a systematic review and meta-analysis. Human Reproduction Update 16 347-363. (doi:10.1093/humupd/dmq001)

Moran LJ, Strauss BJ \& Teede HJ 2011 Diabetes risk score in the diagnostic categories of polycystic ovary syndrome. Fertility and Sterility 95 1742-1748. (doi:10.1016/j.fertnstert.2011.01.133)

Moran LJ, Teede HJ, Noakes M, Clifton PM, Norman RJ \& Wittert GA 2013 Sex hormone binding globulin, but not testosterone, is associated with the metabolic syndrome in overweight and obese women with polycystic ovary syndrome. Journal of Endocrinologial Investigation 36 1004-1010.

Plymate SR, Matej LA, Jones RE \& Friedl KE 1988 Inhibition of sex hormone-binding globulin production in the human hepatoma (Hep G2) cell line by insulin and prolactin. Journal of Clinical Endocrinology and Metabolism 67 460-464. (doi:10.1210/jcem-67-3-460)

Rauschert S, Uhl O, Koletzko B, Kirchberg F, Mori TA, Huang RC, Beilin LJ, Hellmuth C \& Oddy WH 2016 Lipidomics reveals associations of phospholipids with obesity and insulin resistance in young adults. Journal of Clinical Endocrinology and Metabolism 101 871-879. (doi:10.1210/jc.2015-3525)

Scherer M, Montoliu I, Qanadli SD, Collino S, Rezzi S, Kussmann M, Giusti V \& Martin FP 2015 Blood plasma lipidomic signature of epicardial fat in healthy obese women. Obesity 23 130-137. (doi:10.1002/oby.20925)

Schmitz G \& Ruebsaamen K 2010 Metabolism and atherogenic disease association of lysophosphatidylcholine. Atherosclerosis 208 10-18. (doi:10.1016/j.atherosclerosis.2009.05.029)

Shirouchi B, Nagao K, Inoue N, Ohkubo T, Hibino H \& Yanagita T 2007 Effect of dietary omega 3 phosphatidylcholine on obesity-related disorders in obese Otsuka Long-Evans Tokushima fatty rats. Journal of Agricultural and Food Chemistry 55 7170-7176. (doi:10.1021/ jf071225x) 
Stepto NK, Cassar S, Joham AE, Hutchison SK, Harrison CL, Goldstein RF \& Teede HJ 2013 Women with polycystic ovary syndrome have intrinsic insulin resistance on euglycaemic-hyperinsulaemic clamp. Human Reproduction 28 777-784. (doi:10.1093/humrep/des463)

Tonks KT, Coster AC, Christopher MJ, Chaudhuri R, Xu A, GagnonBartsch J, Chisholm DJ, James DE, Meikle PJ, Greenfield JR, et al. 2016 Skeletal muscle and plasma lipidomic signatures of insulin resistance and overweight/obesity in humans. Obesity 24 908-916. (doi:10.1002/oby.21448)

Toulis KA, Goulis DG, Mintziori G, Kintiraki E, Eukarpidis E, Mouratoglou SA, Pavlaki A, Stergianos S, Poulasouchidou M, Tzellos TG, et al. 2011 Meta-analysis of cardiovascular disease risk markers in women with polycystic ovary syndrome. Human Reproduction Update 17 741-760. (doi:10.1093/humupd/dmr025)
Wallner S \& Schmitz G 2011 Plasmalogens the neglected regulatory and scavenging lipid species. Chemistry and Physics of Lipids 164 573-589. (doi:10.1016/j.chemphyslip.2011.06.008)

Weir JM, Wong G, Barlow CK, Greeve MA, Kowalczyk A, Almasy L, Comuzzie AG, Mahaney MC, Jowett JB, Shaw J, et al. 2013 Plasma lipid profiling in a large population-based cohort. Journal of Lipid Research 54 2898-2908. (doi:10.1194/jlr.P035808)

Wild RA, Rizzo M, Clifton S \& Carmina E 2011 Lipid levels in polycystic ovary syndrome: systematic review and meta-analysis. Fertility and Sterility 95 1073-1079.e1071-e1011. (doi:10.1016/j. fertnstert.2010.12.027)

Yang L, Li M, Shan Y, Shen S, Bai Y \& Liu H 2016 Recent advances in lipidomics for disease research. Journal of Separation Science 39 38-50. (doi:10.1002/jssc.201500899)

Received in final form 24 March 2017

Accepted 12 May 2017

Accepted Preprint published online 12 May 2017 http://jme.endocrinology-journals.org DOI: 10.1530/JME-17-0023
() 2017 Society for Endocrinology Printed in Great Britain
Published by Bioscientifica Ltd. 